\title{
Quartz tuning-fork based carbon nanotube transfer into quantum device geometries
}

\author{
S. Blien, ${ }^{1}$ P. Steger ${ }^{1}$ A. Albang, ${ }^{1}$ N. Paradiso, ${ }^{1}$ and A. K. Hüttel ${ }^{1, *}$ \\ ${ }^{1}$ Institute for Experimental and Applied Physics, University of Regensburg, \\ Universitätsstr. 31, 93053 Regensburg, Germany
}

(Dated: 16 May 2018)

\begin{abstract}
With the objective of integrating single clean, as-grown carbon nanotubes into complex circuits, we have developed a technique to grow nanotubes directly on commercially available quartz tuning forks using a high temperature CVD process. Multiple straight and aligned nanotubes bridge the $>100 \mu \mathrm{m}$ gap between the two tips. The nanotubes are then lowered onto contact electrodes, electronically characterized in situ, and subsequently cut loose from the tuning fork using a high current. First quantum transport measurements of the resulting devices at cryogenic temperatures display Coulomb blockade characteristics.
\end{abstract}

\section{INTRODUCTION}

A fabrication technique that has led to many remarkable observations in quantum transport is the in-situ growth of carbon nanotubes onto pre-existing electrodes and trenches in between them [1]. Published results range from Coulomb blockade transport spectroscopy of unperturbed electronic systems [2-5] all the way to high quality factor mechanical resonators and strong interaction between single electron tunneling and vibrational motion [6-10]. A natural limitation of this technique is that the electrode chip is exposed to the conditions of chemical vapour deposition (CVD) nanotube growth, typically $10-30 \mathrm{~min}$ in a gas mixture of hydrogen and methane at $800-1000{ }^{\circ} \mathrm{C}$ [11]. Only few thin film materials survive this process, notably platinum-tungsten combinations $[1,6]$ and rhenium or rhenium-molybdenum alloys [12-15]. Still, fabrication remains challenging and the integration of more sensitive circuit elements such as, e.g., Josephson junctions, quasi impossible.

The separation of growth and measurement chip provides a compelling alternative to in-situ growth of CNTs [16-20]. For the subsequent transfer of the nanotubes from one to the other, several approaches exist. While pressing growth surfaces directly onto the measurement chip to transfer CNTs potentially provides many viable devices per fabrication step and allows the lithographic selection of suitable CNTs on the target surface for contacting [21, 22], the integration of clean, suspended CNTs into complex, large-scale circuits requires a controlled deposition of single macromolecules [18-20].

Here, we present a technique to grow clean CNTs between the two prongs of commercially available quartz tuning forks and subsequently deposit them onto contact electrodes of arbitrary material. We demonstrate the details of the substrates, the transfer, and the cutting process and show first low temperature transport data.

*andreas.huettel@ur.de
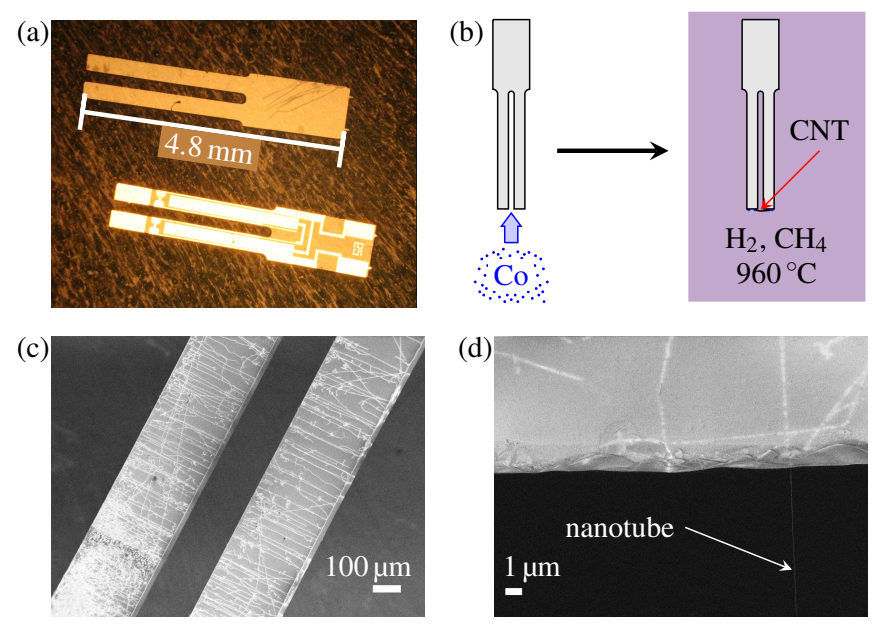

FIG. 1. (a) Commercial quartz tuning forks before and after removal of the metallization. (b) A thin Co layer is sputtered onto the tips of the fork as catalyst for the carbon nanotube growth by chemical vapour deposition. (c) Scanning electron micrograph of a fork after carbon nanotube growth: the nanotubes clearly display a preferred growth direction. For better visibility, here the entire fork surface has been covered with Co growth catalyst. (d) Scanning electron micrograph of a carbon nanotube crossing the gap between the two fork tips.

\section{CNT GROWTH ON QUARTZ TUNING FORKS}

We start with a wafer piece containing several commercial-grade quartz tuning forks, see Fig. 1(a). After breaking out one or more forks, the metallic contacts are removed using aqua regia, hot hydrochloric acid and hot $\mathrm{NaOH}$ baths and successive cleaning steps of sonication and plasma ashing. Then, a nominally $1 \mathrm{~nm}$ thick layer of cobalt is sputter-deposited onto the tips of a fork, see Fig. 1(b). For such a nominal thickness Co does not form a homogeneous film, but a randomly distributed ensemble of Co clusters which serve as catalyst centers for the carbon nanotube growth $[23,24]$.

As next step, the forks are placed on a glass plate and inserted into the quartz tube of a CVD furnace. The furnace is heated up under a steady flow of an argon / 

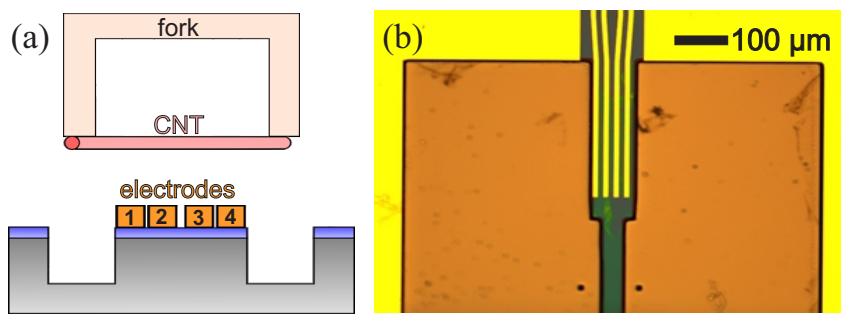

FIG. 2. (a) Schematic of the carbon nanotube transfer: the fork carrying a nanotube is sunk into two trenches that are locally etched into a target chip on both sides of four gold electrodes. (b) Optical micrograph of the target chip: four contact electrodes and a ground plane (yellow), the elevated center ridge carrying the electrodes (dark green), and surrounding deep-etched areas (orange) are visible.

hydrogen mixture and then kept at $960^{\circ} \mathrm{C}$ for 30 minutes under a constant gas flow of methane and hydrogen. The flow rates, $10 \mathrm{sccm} \mathrm{CH}_{4}$ and $20 \mathrm{sccm} \mathrm{H}_{2}$, are typical for clean CNT growth [11]. The fork is placed perpendicular to the gas stream. As a result, the growth is directional in the sense that CNTs grow mainly in the prong-toprong direction, see Fig. 1(b) and also Fig. 1(c,d), where the entire fork surface has been covered with catalyst for better visibility of the resulting nanotube growth.

Imaging the forks in a scanning electron microscope after growth, we find that even with catalyst coating only the fork tips typically up to five nanotubes or nanotube bundles per fork are suspended over the gap between the tips $[5,25]$. To avoid damage and carbon contamination, we do not image forks that are actually used for transfer. In a future setup one could imagine using optical means, as, e.g., Raman or photoluminescence imaging [26] to count the suspended nanotubes between the fork prongs.

\section{TARGET CHIP}

For first tests of the transfer process, devices with four long electrodes were prepared via optical lithography, see Fig. 2(a) for a schematic side view and Fig. 2(b) for a microscope top view. The substrate is highly pdoped silicon, with a $500 \mathrm{~nm}$ thermally grown surface oxide. On its surface, four finger-like gold electrodes are deposited using thermal evaporation, and lift-off. The typical width of the electrodes and the distance between them are both $10 \mu \mathrm{m}$ for this simplified test device. Next to the electrodes, two rectangular areas are locally etched to a depth of $12 \mu \mathrm{m}$ by an anisotropic reactive ion etching process using $\mathrm{SF}_{6}$ and Ar. The etch depth should be as large as possible and is mainly limited by the lithographic resist protecting the remaining structure.
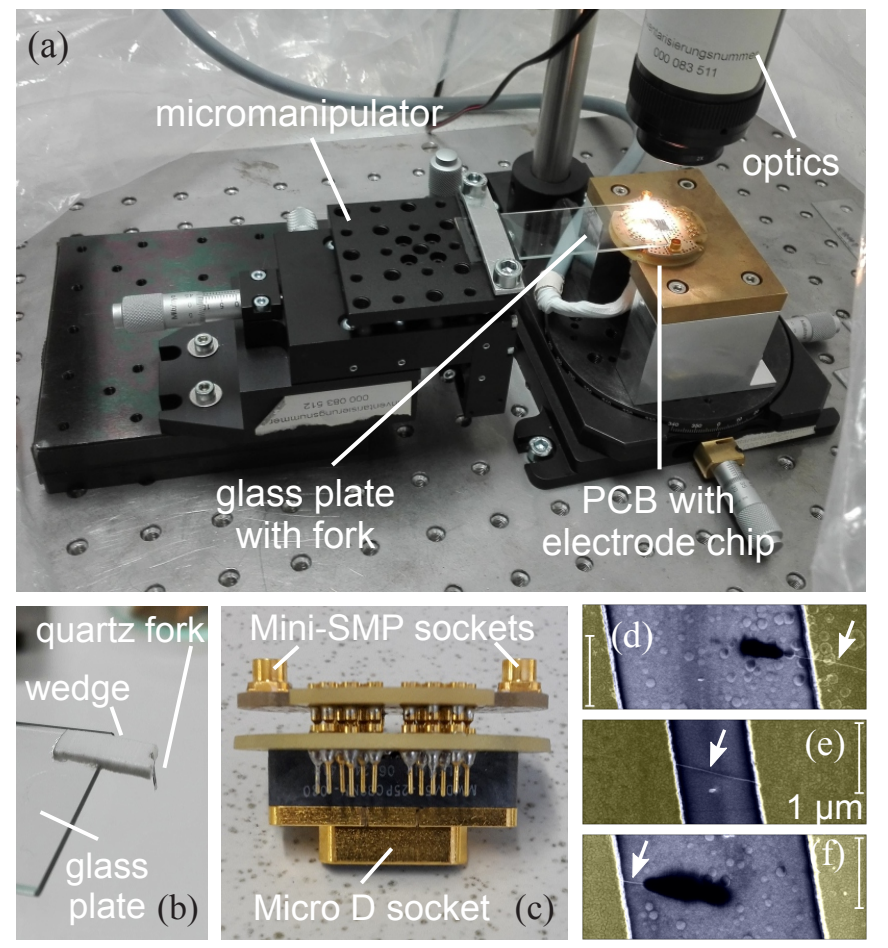

FIG. 3. (a) Transfer setup: the quartz fork is mounted on a micromanipulator stage. It can be lowered to the target chip, which is glued onto a printed circuit board (PCB) and is electrically connected. The process is monitored via an optical microscope with a zoom lens and a camera. (b) Detail picture of how the quartz fork is mounted on the glass plate. (c) Side view of the sample holder: to establish connection to electronic devices, a second PCB with a Micro D socket is attached. For further experiments, two high frequency ports with Mini-SMP connectors are additionally soldered on top of the board. (d-f) Scanning electron micrographs of a successfully transferred CNT: the nanotube has been cut between each pair of outer electrodes $(d, f)$ and now only connects the two inner electrodes (e).

\section{TRANSFER AND CUTTING PROCESS}

For the transfer, the quartz fork carrying as-grown CNTs is attached to a glass object plate and mounted on a micromanipulator stage, see Figs. 3(a) and (b). The setup is adapted from the equipment combination used in [27] to dry-stamp 2D materials. As there, a camera combined with a zoom lens allows us to observe the target chip from the top. The base plate is modified insofar as it clamps a printed circuit board sample holder with a 25-pin MDM socket at the bottom, see Fig. 3(c). The target chip is glued onto the circuit board and bonded; the electrodes are electrically contacted during the transfer process.

Using the micromanipulator stage, the quartz fork is lowered onto the chip such that its tips sink into the deep-etched areas on both sides of the dc contacts, cf. Fig. 2(a). The process is monitored both optically and electrically. On the one hand, we use the microscope 

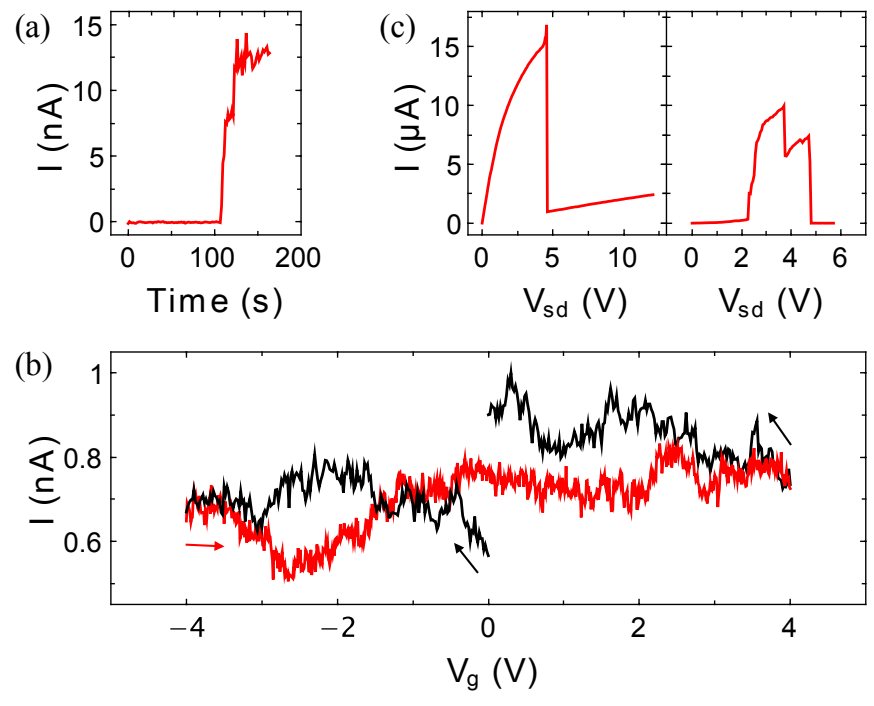

FIG. 4. (a) The current between the voltage biased contacts 1 and 4, see Fig. 2(a), is measured continuously while a quartz fork is lowered onto the target chip. As soon as a CNT touches the electrodes a finite current can flow. (b) Example back gate voltage sweep at a bias voltage of $3 \mathrm{mV}$, recorded during a transfer process before cutting the nanotube. This allows estimating the type (metallic, semiconducting or bundle) of nanotube before finally leaving it on the device. (c) Current measured during two different voltage ramps for "cutting" a CNT. From the shape of the resulting curves one can draw conclusions on the transfer result, see the text.

camera to monitor the fork position during the alignment. On the other hand, by applying $100 \mathrm{mV}$ between contacts 1 and 4, see Fig. 2(a), we can detect a CNT bridging the metal electrodes by simply measuring a finite current. This is illustrated in Fig. 4(a), where at a time index of $t \approx 110 \mathrm{~s}$ contact is made. Back gate voltage sweeps, see Fig. 4(b), then allow us to estimate whether a semiconducting or metallic nanotube or a nanotube bundle is contacted.

By ramping up a voltage bias and thereby the current between contacts 1 and 2, as well as subsequently between contacts 3 and 4 , while the device is in air, the segments of the tube between these contacts can be electrically cut. Example current-voltage characteristics during this process are plotted in Fig. 4(c). The critical current for cutting a nanotube typically lies in the range of $10-30 \mu \mathrm{A}$, consistent with the findings of Refs. [19, 20]. If at a certain point the current drops to zero in one single step as, e.g., in the left part of Fig. 4(c), this indicates that one single-wall carbon nanotube has been cut. If the current decreases to zero in several steps as in the right part of Fig. 4(c) the segment was a multi-wall nanotube or bundle and the steps correspond to breaking the shells or nanotubes one at a time. We were able to verify this interpretation of the number of steps in the I-V-curves by extracting the diameter of successfully transferred nanotubes from atomic force microscopy images at large contact distances, where the nanotubes can touch the substrate.

If the approach of fork and target chip is not done carefully enough, a nanotube can be ripped off the fork tips and then fall down to the substrate in the deep-etched areas. Then, electrodes 1 and 2 are still electrically connected via the substrate even after the nanotube segment between them has been cut, resulting in a tail of finite current in the I-V-curve, cf. Fig.4(c), left panel.

\section{CLEANING OF THE QUARTZ FORKS FOR RE-USE}

After successful completion of the cutting process the detached nanotube lies only over the inner contact pair (2 and 3), as can be seen in the SEM image of Fig. 3(e). The quartz fork can then be safely lifted and removed.

Given the chemical and mechanical stability of the tuning forks, a rigorous cleaning procedure can subsequently be applied to remove both carbon residues and cobalt catalyst. We use plasma ashing to remove organic compounds grown in the preceding CVD process, and a bath of hot nitric acid to dissolve residues of old catalyst. After sonication and another short plasma ashing step the forks can be reintroduced into the fabrication cycle by sputtering a new layer of Co catalyst.

\section{LOW TEMPERATURE CHARACTERIZATION}

After successfully transferring a carbon nanotube to a substrate similar to the one shown in Fig. 2, we have cooled down the device to liquid helium temperature. The device was fabricated on a highly doped Si wafer, such that the substrate can be electrically connected and used as a global backgate. Fig. 5(a) shows the current through the CNT in dependence on the gate voltage $V_{\mathrm{g}}$, when $2 \mathrm{mV}$ bias is applied. Several distinct gate voltage regions can be distinguished in the figure. For $V_{\mathrm{g}}<1.8 \mathrm{~V}$ the nanotube is strongly coupled to the electrodes, resulting in an open system. In the region $1.8 \mathrm{~V}<V_{\mathrm{g}}<2.8 \mathrm{~V}$ Coulomb blockade and single electron tunneling peaks are visible; see Fig. 5(b) for a detail zoom. For $2.8 \mathrm{~V}<V_{\mathrm{g}}$ no current is flowing, indicating an electronic band gap.

A stability diagram at millikelvin temperatures of a similar device, where a carbon nanotube was deposited as described here, is shown in Fig. 5(c). The figure displays the differential conductance as function of the sourcedrain voltage $V_{\text {sd }}$ and a gate voltage $V_{\mathrm{g}}$. One can clearly identify the characteristic diamond pattern of Coulomb blockade regions as typically shown by quantum dots.

The stability diagram of Fig. 5(c) indicates a predominant electrostatic charging energy of approximately $E_{c}=0.3 \mathrm{meV}$, corresponding to a total quantum dot capacitance of $C_{\Sigma}=e^{2} / E_{c}=530 \mathrm{aF}$. This is significantly larger than typical values for a device with single-wall nanotube length $l=1.4 \mu \mathrm{m}$ and a distance to the gate 


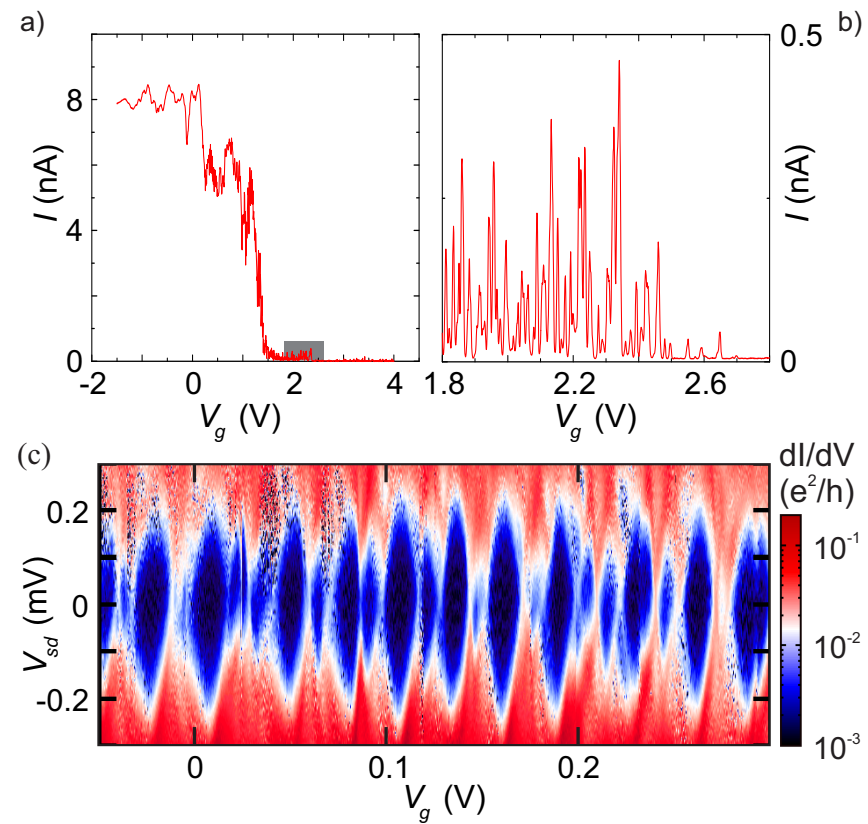

FIG. 5. (a) Characterization of a transferred CNT at $T=4.2 \mathrm{~K}$. Plotted is the current through the nanotube as a function of the gate voltage $V_{\mathrm{g}}$, at an applied source-drain voltage of $2 \mathrm{mV}$. Different parameter regions can be distinguished, see the text. (b) Zoom into the shaded area of (a), displaying Coulomb oscillations of the current. (c) Stability diagram of a transferred CNT at $T=15 \mathrm{mK}$; differential conductance as function of gate voltage and source-drain voltage. A pattern of Coulomb blockade areas with two distinct sizes is visible.

of $d=500 \mathrm{~nm}$, the values expected from the contact geometry here. The small charging energy may indicate that multiwall nanotubes, bundles or nanotube networks have been transferred and measured. The appearance of an additional set of smaller Coulomb blockade areas in Fig. 5(c) supports this, indicating a second confined electronic system. No transversal mechanical resonance was found in transport measurements in a frequency range of $100 \mathrm{kHz} \leq f_{\text {drive }} \leq 500 \mathrm{MHz}$ [6]. Further optimiza- tion of the CVD parameters and the transfer procedure to produce solitary single-wall carbon nanotubes is thus required.

\section{CONCLUSIONS AND OUTLOOK}

We have implemented a technique for carbon nanotube transfer separating growth and measurement onto different substrates. Nanotubes are grown on the tips of commercially available quartz tuning forks and subsequently transferred to a target chip of desired design.

As with other nanotube transfer procedures, the choice of contact materials and circuit elements for the target chip is much less constrained than for in situ overgrowth, carbon nanotubes not suitable for measurements can easily be removed, and complex-structured devices can be re-used in more than one transfer attempt. Transfer targets may range from, e.g., superconducting coplanar circuit geometries [28-30], qubit circuits [31], superconducting single electron transistors [32, 33], or ferromagnetic contact electrodes [34], all the way to diamond crystallites containing NV-centers [35].

The quartz tuning forks are standardized, macroscopic parts that can be obtained in large numbers. In addition, they are highly robust, and survive multiple cycles of catalyst deposition, growth, nanotube transfer, and cleaning. This allows an easy, systematic approach towards integrating carbon nanotubes into devices of arbitrary complexity.

\section{ACKNOWLEDGMENTS}

The authors thank E. Weig for the initial suggestion of using quartz tuning forks, and Coftech $\mathrm{GmbH}$ for the quartz tuning fork wafer. Transport data has been recorded using the Lab::Measurement software package [36]. We acknowledge funding by the Deutsche Forschungsgemeinschaft via grants Hu 1808/1, SFB 689, and GRK 1570.
[1] J. Cao, Q. Wang, and H. Dai, "Electron transport in very clean, as-grown suspended carbon nanotubes," Nature Materials 4, 745-749 (2005).

[2] V. V. Deshpande and M. Bockrath, "The onedimensional Wigner crystal in carbon nanotubes," Nature Physics 4, 314-318 (2008).

[3] F. Kuemmeth, S. Ilani, D. C. Ralph, and P. L. McEuen, "Coupling of spin and orbital motion of electrons in carbon nanotubes," Nature 452, 448-452 (2008).

[4] S. Pecker, F. Kuemmeth, A. Secchi, M. Rontani, D. C. Ralph, P.L. McEuen, and S. Ilani, "Observation and spectroscopy of a two-electron Wigner molecule in an ultraclean carbon nanotube," Nat. Phys. 9, 576 (2013).
[5] M. Marganska, D. R. Schmid, P. L. Stiller, A. Dirnaichner, Ch. Strunk, M. Grifoni, and A. K. Hüttel, "Shaping electron wave functions in a carbon nanotube with a parallel magnetic field," (2017), arXiv:1712.08545.

[6] A. K. Hüttel, G. A. Steele, B. Witkamp, M. Poot, L. P. Kouwenhoven, and H. S. J. van der Zant, "Carbon nanotubes as ultra-high quality factor mechanical resonators," Nano Letters 9, 2547-2552 (2009).

[7] G. A. Steele, A. K. Hüttel, B. Witkamp, M. Poot, H. B. Meerwaldt, L. P. Kouwenhoven, and H. S. J. van der Zant, "Strong coupling between single-electron tunneling and nanomechanical motion," Science 325, 1103 (2009).

[8] B. Lassagne, Y. Tarakanov, J. Kinaret, D. GarciaSanchez, and A. Bachtold, "Coupling mechanics to 
charge transport in carbon nanotube mechanical resonators," Science 28, 1107 (2009).

[9] A. K. Hüttel, H. B. Meerwaldt, G. A. Steele, M. Poot, B. Witkamp, L. P. Kouwenhoven, and H. S. J. van der Zant, "Single electron tunneling through high-Q singlewall carbon nanotube NEMS resonators," phys. stat. sol. (b) 247, 2974 (2010).

[10] K. J. G. Götz, D. R. Schmid, F. J. Schupp, P. L. Stiller, Ch. Strunk, and A. K. Hüttel, "Nanomechanical characterization of the Kondo charge dynamics in a carbon nanotube," (2018), arXiv:1802.00522.

[11] J. Kong, H. T. Soh, A. M. Cassell, C. F. Quate, and H. Dai, "Synthesis of individual single-walled carbon nanotubes on patterned silicon wafers," Nature 395, 878 (1998).

[12] V. Singh, B. H. Schneider, S. J. Bosman, E. P. J. Merkx, and G. A. Steele, "Molybdenum-rhenium alloy based high- $Q$ superconducting mircowave resonators," Applied Physics Letters 105, 222601 (2014).

[13] D. R. Schmid, S. Smirnov, M. Margańska, A. Dirnaichner, P. L. Stiller, M. Grifoni, A. K. Hüttel, and C. Strunk, "Broken SU(4) symmetry in a Kondocorrelated carbon nanotube," Physical Review B 91, 155435 (2015).

[14] K. J. G. Götz, S. Blien, P. L. Stiller, O. Vavra, T. Mayer, T. Huber, T. N. G. Meier, M. Kronseder, Ch. Strunk, and A. K. Hüttel, Nanotechnology 27, 135202 (2016).

[15] S. Blien, K. J. G. Götz, P. L. Stiller, T. Mayer, T. Huber, O. Vavra, and A. K. Hüttel, "Towards carbon nanotube growth into superconducting microwave resonator geometries," phys. stat. sol. (b) 253, 2385 (2016).

[16] Chung Chiang Wu, Chang Hua Liu, and Zhaohui Zhong, "One-step direct transfer of pristine single-walled carbon nanotubes for functional nanoelectronics," Nano Letters 10, 1032-1036 (2010).

[17] Fei Pei, Edward A. Laird, Gary A. Steele, and Leo P. Kouwenhoven, "Valley-spin blockade and spin resonance in carbon nanotubes," Nature Nanotechnology 7, 630634 (2012).

[18] V. Ranjan, G. Puebla-Hellmann, M. Jung, T. Hasler, A. Nunnenkamp, M. Muoth, C. Hierold, A. Wallraff, and C. Schönenberger, "Clean carbon nanotubes coupled to superconducting impedance-matching circuits," Nature Communications 6, 7165 (2015).

[19] J. Waissman, M. Honig, S. Pecker, A. Benyamini, A. Hamo, and S. Ilani, "Realization of pristine and locally tunable one-dimensional electron systems in carbon nanotubes," Nature Nanotechnology 8, 569 (2013).

[20] Jörg Gramich, Andreas Baumgartner, Matthias Muoth, Christofer Hierold, and Christian Schönenberger, "Fork stamping of pristine carbon nanotubes onto ferromagnetic contacts for spin-valve devices," physica status solidi (b) 252, 2496-2502 (2015).

[21] M. M. Desjardins, J. J. Viennot, M. C. Dartiailh, L. E. Bruhat, M. R. Delbecq, M. Lee, M.-S. Choi, A. Cottet, and T. Kontos, "Observation of the frozen charge of a Kondo resonance," Nature 545, 4 (2017).

[22] N. Paradiso et al., (2018), in preparation.

[23] Mukul Kumar and Yoshinori Ando, "Chemical vapor deposition of carbon nanotubes: a review on growth mech- anism and mass production," Journal of Nanoscience and Nanotechnology 10, 3739-3758 (2010).

[24] Dongning Yuan, Lei Ding, Haibin Chu, Yiyu Feng, Thomas P. McNicholas, and Jie Liu, "Horizontally aligned single-walled carbon nanotube on quartz from a large variety of metal catalysts," Nano Letters 8, 25762579 (2008).

[25] Y. A. Kasumov, A. Shailos, I. I. Khodos, V. T. Volkov, V. I. Levashov, V. N. Matveev, S. Guéron, M. Kobylko, M. Kociak, H. Bouchiat, V. Agache, A. S. Rollier, L. Buchaillot, A. M. Bonnot, and A. Y. Kasumov, "CVD growth of carbon nanotubes at very low pressure of acetylene," Applied Physics A 88, 687-691 (2007).

[26] Jacques Lefebvre, David G. Austing, Jeffery Bond, and Paul Finnie, "Photoluminescence imaging of suspended single-walled carbon nanotubes," Nano Letters 6, 1603 1608 (2006).

[27] Andres Castellanos-Gomez, Michele Buscema, Rianda Molenaar, Vibhor Singh, Laurens Janssen, Herre S J van der Zant, and Gary A Steele, "Deterministic transfer of two-dimensional materials by all-dry viscoelastic stamping," 2D Materials 1, 011002 (2014).

[28] Ze-Liang Xiang, Sahel Ashhab, J. Q. You, and Franco Nori, "Hybrid quantum circuits: Superconducting circuits interacting with other quantum systems," Rev. Mod. Phys. 85, 623-653 (2013).

[29] Markus Aspelmeyer, Tobias J. Kippenberg, and Florian Marquardt, "Cavity optomechanics," Rev. Mod. Phys. 86, 1391-1452 (2014).

[30] Xiu Gu, Anton Frisk Kockum, Adam Miranowicz, Yu-Xi Liu, and Franco Nori, "Microwave photonics with superconducting quantum circuits," Physics Reports 718-719, 1 - 102 (2017).

[31] Xin Wang, Adam Miranowicz, Hong-Rong Li, and Franco Nori, "Hybrid quantum device with a carbon nanotube and a flux qubit for dissipative quantum engineering," Phys. Rev. B 95, 205415 (2017).

[32] András Pályi, P. R. Struck, Mark Rudner, Karsten Flensberg, and Guido Burkard, "Spin-orbit-induced strong coupling of a single spin to a nanomechanical resonator," Phys. Rev. Lett. 108, 206811 (2012).

[33] Philipp R. Struck, Heng Wang, and Guido Burkard, "Nanomechanical readout of a single spin," Phys. Rev. B 89, 045404 (2014).

[34] P. Stadler, W. Belzig, and G. Rastelli, "Control of vibrational states by spin-polarized transport in a carbon nanotube resonator," Phys. Rev. B 91, 085432 (2015).

[35] Peng-Bo Li, Ze-Liang Xiang, Peter Rabl, and Franco Nori, "Hybrid quantum device with nitrogen-vacancy centers in diamond coupled to carbon nanotubes," Phys. Rev. Lett. 117, 015502 (2016).

[36] S. Reinhardt, C. Butschkow, S. Geissler, A. Dirnaichner, F. Olbrich, C. Lane, D. Schröer, and A. K. Hüttel, "Lab::Measurement - a portable and extensible framework for controlling lab equipment and conducting measurements," arXiv preprint (2018), arXiv:1804.03321. 